$\mathbb{T}$ periodica polytechnica

Mechanical Engineering

$51 / 2(2007) 89-97$

doi: 10.3311/pp.me.2007-2.08

web: http://www.pp.bme.hu/me

(c) Periodica Polytechnica 2007

RESEARCH ARTICLE

\section{Application of prestressed transparent composite beams in fracture mechanics}

\author{
András Szekrényes
}

Received 2008-01-18

\begin{abstract}
This work presents the mixed-mode I/II/III version of the prestressed end-notched flexure system for the general delamination characterization of composite materials. The novel fracture mechanical configuration combines the traditional modeI double-cantilever beam and the mode-II end-notched flexure specimens with the mode-III modified split-cantilever beam. First, a steel roller with a given diameter - which should not exceed the critical crack opening - is inserted to the delamination front, this fixes the mode-I part of the total energy release rate. Second, the prestressed specimen is put into the special rig of the MSCB specimen, and with the help of a prestressing screw the mode-III part of the total energy release rate is also fixed. Third, the prestressed specimen is put into a simple three-point bending setup and the mode-II part of the total energy release rate is increased up to fracture initiation. Using this method, i.e. varying the crack opening displacement by the steel roller and the crack tearing displacement by the MSCB rig the fracture surface in the $G_{I}-G_{I I}-G_{I I I}$ space can be obtained. To demonstrate the applicability and limitations of the novel system experiments on glass-fiber reinforced polyester specimens were performed including separated mode-I, mode-II, mode-III, mixed-mode I/II, II/III, I/III and I/II/III fracture tests. To reduce the measured data a previously validated improved beam theory scheme is applied. Finally, the surface of the fracture criterion is constructed by the generalization of the traditional criterion by Williams.
\end{abstract}

\section{Keywords}

composite $\cdot$ variable mode-mixity $\cdot$ beam theory $\cdot$ interlaminar fracture

\section{András Szekrényes}

Department of Applied Mechanics, BME, H-1111 Budapest, Múegyetem rkp. 5., Hungary

e-mail: szeki@mm.bme.hu

\section{Introduction}

The delamination testing of composite materials is extremely important due to the low interlaminar strength. Considering the linear elastic fracture mechanics (LEFM) there are three basic types of fracture modes: mode-I (opening), mode-II (inplane shear or sliding), mode-III (anti-plane shear or tearing) [1]. In the last thirty years a large number of excellent test methods were developed including the mode-I double-cantilever beam (DCB) [2], the mode-II end-notched flexure (ENF) [3], the mode-III edge crack torsion (ECT) [4] and - among others - the mixed-mode bending (MMB, mixed-mode I/II) [5] specimens. Considering these systems a large amount of experimental result was presented in the composite literature. For more details on the available fracture mechanical systems and specimen types refer to $[6,7]$. From other perspectives significantly less work was proposed for the mixed-mode I/II, II/III and I/II/III fractures. Although there are some papers dealing with the combined modes [8-10], these works present experiments performed mainly on different types of metals and apply the usual compact tension (CT) specimen with an inclined crack. In composite materials the traditional beam-like specimen is more likely to be preferred. This motivated the author to develop a universal method which could make it possible to test the material under mixed-mode I/II/III loading using beam-like specimens.

The application of prestressed beams in composite fracture mechanics was introduced by the present author. In a recent paper [11] the mixed-mode I/II version of the prestressed endnotched flexure $\left(\mathrm{PENF}_{I / I I}\right)$ coupon was presented. A steel roller was inserted to the delamination front of a unidirectional beam-like (DCB) specimen, which caused a fixed crack opening displacement. Putting this prestressed DCB specimen into a three-point bending setup the applied load can be increased up to fracture initiation. This system produces mixed-mode I/II fracture in a very simple way, although it involves several drawbacks, namely: the mode ratio changes with the crack length and the applied load. Thus, the mode ratio can not be designated before the test. In spite of that the complete range of mode-mixity can be covered. Also, the point of crack initiation should be 
identified with high accuracy; therefore at the present stage the $\mathrm{PENF}_{I / I I}$ specimen is applicable mainly for transparent composite materials.

The next step was to extend the method for mixed-mode II/III delamination fracture. This involved the combination of a modeII and a mode-III specimen. It should be mentioned that only those specimen types can be combined which incorporate the same specimen geometry. In this point of view the only candidates are the mode-II ENF and the mode-III modified splitcantilever beam (MSCB) systems. In a more recent work [12] the mixed-mode II/III PENF (denoted as $\mathrm{PENF}_{I I / I I I}$ ) specimen was developed and a fracture envelope in the $\mathrm{G}_{I I}-\mathrm{G}_{I I I}$ plane was determined. The mode-III energy release rate in unidirectional glass/polyester specimens were fixed by using a special rig, which was originally developed by Sharif et al. [13] and Cicci et al. [14]. Then the prestressed specimen was put into a three-point bending setup (which was the same as that used for the common ENF test) and was loaded up to fracture initiation. The applicability and limitations of the $\mathrm{PENF}_{I I / I I I}$ system was demonstrated using unidirectional glass/polyester specimens and the experimental data was reduced by three techniques. It has been found that a reduction technique based on improved beam theory (IBT) had the sufficient accuracy and simplicity.

Recently the original concept was extended for the mixedmode I/III delamination fracture. Based on the former works the combination of the mode-I DCB and the mode-III MSCB specimen is necessary. First, a steel roller was inserted between the specimen arms of the DCB, second the prestressed specimen was put into the special rig of the MSCB configuration leading to mixed-mode I/III condition. The new configuration (denoted as prestressed split-cantilever beam - $\operatorname{PSCB}_{I / I I I}$ ) was analysed using beam and finite element models, respectively. Furthermore experiments on glass/polyester composite specimens were also performed. The measured data is reduced by three techniques: improved beam theory, finite element method and the experimental compliance calibration method, respectively. Based on the experiments the fracture envelope in the $\mathrm{G}_{I}-\mathrm{G}_{I I I}$ plane was constructed for the glass/polyester material. An important conclusion is that there is a significant interaction between the mode-I and mode-III ERRs. It is also important to note that in each case there is a little mode-II contribution to the total energy release rate, however it can be reduced to $2-5 \%$ depending on the ratio of $\mathrm{G}_{I}$ and $\mathrm{G}_{I I I}$. Finally the obtained fracture envelope is compared to those in the $\mathrm{G}_{I}-\mathrm{G}_{I I}$ and the $\mathrm{G}_{I I}-\mathrm{G}_{I I I}$ plane. The construction of the relevant paper is in progress [15].

The main object of this work is the application of the original concept and to construct a general mixed-mode I/II/III system for the fracture characterization of composite materials. We simply combine the experimental equipment of the previous systems: we apply a steel roller to fix the mode-I part of the ERR and the special rig to fix the mode-III part of it. Then we put the double prestressed composite specimen into a simple threepoint bending setup and vary the values of the prestressing displacements. This way the complete $\mathrm{G}_{I}-\mathrm{G}_{I I}-\mathrm{G}_{I I I}$ space can be investigated.

\section{Improved beam theory for data reduction}

Based on the geometry of the specimens (Fig. 1) we can treat them as elastic beams. In accordance with previous works $[12,15]$ the compliance and the energy release rate (ERR) expressions for the DCB, ENF and MSCB coupons are known with the sufficient accuracy. The $\mathrm{PENF}_{I / I I / I I I}$ is in fact the superposition of these specimens, and consequently, it is reasonable to assume that the analytical solutions can also be applied to the combination of them. So, first we present the expressions for the DCB specimen (Fig. 1a). The compliance is:

$$
C^{D C B}=\frac{8 a^{3}}{b h^{3} E_{11}}+\frac{2 a^{3}}{b h^{3} E_{11}}\left(f_{W 1}+f_{T}+\frac{f_{S V}}{2}\right),
$$

where $a$ is the crack length, $b$ is the width of the specimen, $h$ is the half thickness, $E_{11}$ is the flexural modulus of the material, while the factors denoted by $f$ are related to the elastic foundation, transverse shear and the so-called Saint-Venant deformations:

$$
\begin{aligned}
f_{W 1}= & 5.07\left(\frac{h}{a}\right)\left(\frac{E_{11}}{E_{33}}\right)^{\frac{1}{4}}+8.58\left(\frac{h}{a}\right)^{2}\left(\frac{E_{11}}{E_{33}}\right)^{\frac{1}{2}}+ \\
& 2.08\left(\frac{h}{a}\right)^{3}\left(\frac{E_{11}}{E_{33}}\right)^{\frac{3}{4}} \\
f_{T}= & \frac{1}{k}\left(\frac{h}{a}\right)^{2}\left(\frac{E_{11}}{G_{13}}\right), \\
f_{S V}= & \frac{12}{\pi}\left(\frac{h}{a}\right)\left(\frac{E_{11}}{G_{13}}\right)^{\frac{1}{2}} .
\end{aligned}
$$

The critical energy release rate of the specimens can be calculated by the following (Irwin-Kies) expression [1]:

$$
G_{C}=\frac{P^{2}}{2 b} \frac{d C}{d a}
$$

so we have that:

$$
G_{I}=\frac{P_{D C B}^{2} a^{2}\left(12+f_{W 2}+f_{T}+f_{S V}\right)}{b^{2} h^{3} E_{11}}
$$

where the factor denoted by $f_{W 2}$ is:

$$
f_{W 2}=10.14\left(\frac{h}{a}\right)\left(\frac{E_{11}}{E_{33}}\right)^{\frac{1}{4}}+8.58\left(\frac{h}{a}\right)^{2}\left(\frac{E_{11}}{E_{33}}\right)^{\frac{1}{2}} .
$$

We express the force in the DCB specimen if there is a fixed crack opening:

$$
P_{D C B}=\frac{b h^{3} E_{11} \delta_{D C B}}{8 a^{3}} \frac{1}{1+\left(f_{W 1}+f_{T}+f_{S V} / 2\right) / 4} .
$$

Substituting it back to Eq. (6) we obtain that:

$$
G_{I}=\frac{h^{3} E_{11} \delta_{D C B}^{2}}{64 a^{4}} \frac{\left[12+f_{W 2}+f_{T}+f_{S V}\right]}{\left[1+\left(f_{W 1}+f_{T}+f_{S V} / 2\right) / 4\right]^{2}},
$$


a.

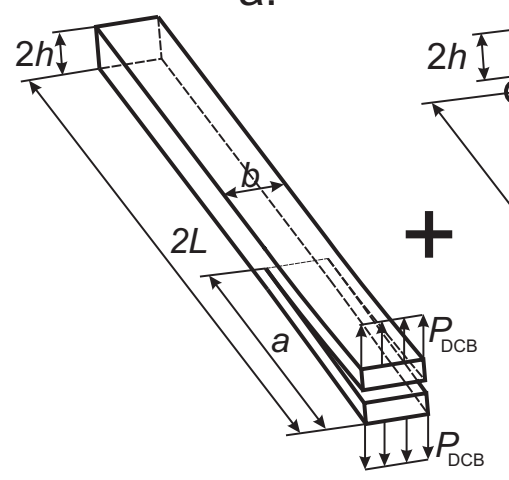

b.

C. d.

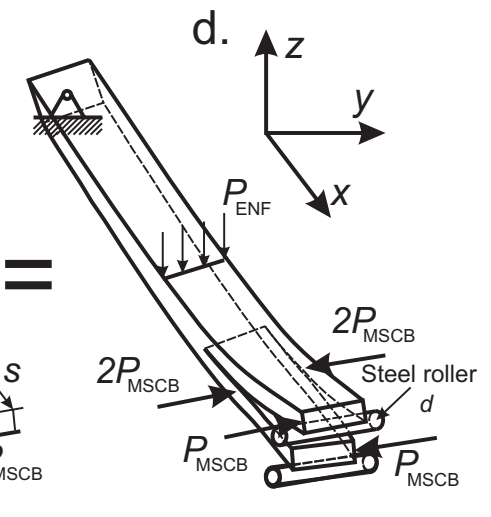

Fig. 1. The mixed-mode I/II/III PENF specimen (d) as the superposition of the DCB (a), ENF (b) and MSCB (c) specimens

where $\delta_{D C B}$ is the crack opening displacement in the DCB specimen. If we use a steel roller to prestress the DCB specimen then $\delta_{D C B}$ is equal to the diameter of the roller.

Now we define the compliance and the ERR for the ENF specimen (Fig. 1b):

$$
C_{E N F}=\frac{3 a^{3}+2 L^{3}}{8 b h^{3} E_{11}}+\frac{2 L}{8 b h k G_{13}}+\frac{a^{3}}{8 b h^{3} E_{11}} f_{S H 1},
$$

where $L$ is the length of the specimen, $k=5 / 6$ is the shear correction factor and $G_{13}$ is the shear modulus of the material in the $x-z$ plane (refer to Fig. 1). The factor is defined as:

$$
f_{S H 1}=0.98\left(\frac{h}{a}\right)\left(\frac{E_{11}}{G_{13}}\right)^{\frac{1}{2}}+0.43\left(\frac{h}{a}\right)^{2}\left(\frac{E_{11}}{G_{13}}\right) .
$$

Using Eq.(5) the ERR of the ENF specimen becomes:

$$
G_{I I}=\frac{P_{E N F}^{2} a^{2}}{16 b^{2} h^{3} E_{11}}\left[9+f_{S H 2}\right]
$$

and we have that:

$$
f_{S H 2}=1.96\left(\frac{h}{a}\right)\left(\frac{E_{11}}{G_{13}}\right)^{\frac{1}{2}}+0.43\left(\frac{h}{a}\right)^{2}\left(\frac{E_{11}}{G_{13}}\right) .
$$
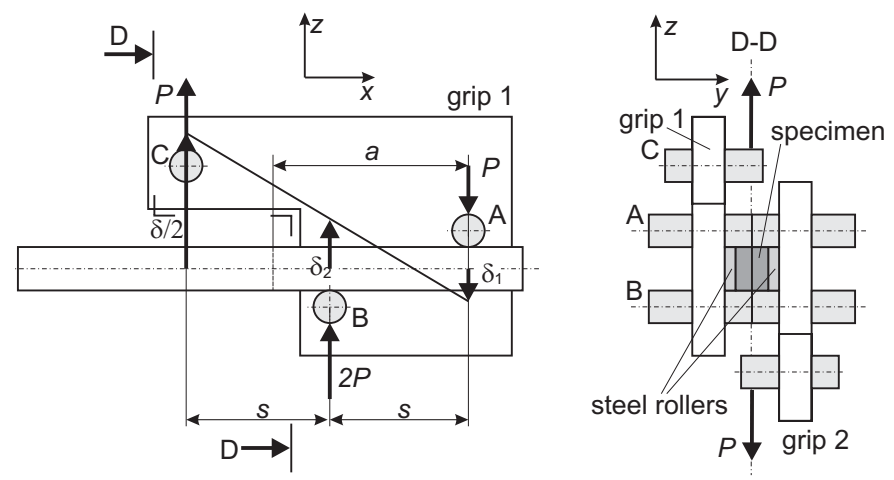

Fig. 2. The views of the mode III MSCB specimen

Finally we need the compliance and the ERR of the mode-III MSCB specimen [12](Fig. 1c). The 2D views can be seen in

Fig. 2. The rollers A and B transfer a scissor-like load to the specimen arms. A detailed analysis of the MSCB specimen is given in [12]. The compliance of the MSCB specimen is:

$$
C_{M S C B}=\frac{8 a^{3}}{b^{3} h E_{11}}\left[f_{E B 1}+f_{T I M 1}+f_{F T 1}+f_{S-V 1}\right],
$$

where:

$$
\begin{aligned}
f_{E B 1} & =1-6\left(\frac{s}{a}\right)+12\left(\frac{s}{a}\right)^{2}-6\left(\frac{s}{a}\right)^{3}, \\
f_{T I M 1} & =0.3\left(\frac{b}{a}\right)^{2}\left(\frac{E_{11}}{G_{13}}\right), \\
f_{F T 1} & =0.19 \frac{1}{\varsigma}\left(\frac{b}{a}\right)^{2}\left(\frac{E_{11}}{G_{12}}\right), \\
f_{S-V 1} & =\left[0.48-1.91\left(\frac{s}{a}\right)+1.91\left(\frac{s}{a}\right)^{2}\right]\left(\frac{b}{a}\right)\left(\frac{E_{11}}{G_{13}}\right)^{\frac{1}{2}},
\end{aligned}
$$

and

$$
\varsigma=1-0.63 \mu \frac{h}{b}, \mu=\left(\frac{G_{13}}{G_{12}}\right)^{\frac{1}{2}},
$$

where $G_{12}$ is the shear modulus of the material in the $x-y$ plane. The ERR is

$$
G_{I I I}^{97.8 \%}=\frac{12 P_{M S C B}^{2} a^{2}}{b^{4} h E_{11}}\left[f_{E B 2}+f_{T I M 2}+f_{F T 2}+f_{S-V 2}\right]
$$

where

$$
\begin{aligned}
f_{E B 2} & =1-4\left(\frac{s}{a}\right)+4\left(\frac{s}{a}\right)^{2}, \\
f_{T I M 2} & =0.1\left(\frac{b}{a}\right)^{2}\left(\frac{E_{11}}{G_{13}}\right), \\
f_{F T 2} & =0.06 \frac{1}{\varsigma}\left(\frac{b}{a}\right)^{2}\left(\frac{E_{11}}{G_{12}}\right), \\
f_{S-V 2} & =\left[0.32-0.64\left(\frac{s}{a}\right)\right]\left(\frac{b}{a}\right)\left(\frac{E_{11}}{G_{13}}\right)^{\frac{1}{2}} .
\end{aligned}
$$

The next step is to express the force in the MSCB specimen

Application of prestressed transparent composite beams in fracture mechanics 
if there is a fixed CTD:

$$
\begin{aligned}
P_{M S C B}= & \frac{b^{3} h E_{11} \delta_{M S C B}}{8 a^{3}} \times \\
& \frac{1}{\left(f_{E B 1}+f_{T I M 1}+f_{F T 1}+f_{S-V 1}\right)} .
\end{aligned}
$$

Substituting it back into Eq. (20) we obtain that

$$
\begin{aligned}
G_{I I I}^{97.8 \%}= & \frac{3}{16} \frac{b^{2} h E_{11} \delta_{M S C B}^{2}}{a^{4}} \times \\
& \frac{\left(f_{E B 2}+f_{T I M 2}+f_{F T 2}+f_{S-V 2}\right)}{\left(f_{E B 1}+f_{T I M 1}+f_{F T 1}+f_{S-V 1}\right)^{2}},
\end{aligned}
$$

where in the superscript the number $97.8 \%$ refers to the optimal case, when the mode-II ERR component is significantly reduced. We need even the mode ratios. Combining the equations above we have that

$$
\begin{aligned}
& \frac{G_{I}}{G_{I I}}= \frac{b^{2} E_{11}^{2}}{3}\left(\frac{h}{a}\right)^{6}\left(\frac{\delta_{D C B}}{P_{E N F}}\right)^{2} \times \\
& \frac{12+f_{W 2}+f_{T}+f_{S V}}{\left[1+\left(f_{w 1}+f_{T}+f_{S V} / 2\right) / 4\right]^{2}} \frac{1}{\left(9+f_{S H 2}\right)}, \\
& \frac{G_{I I I}^{97.8 \%}}{G_{I I}}= \frac{3 b^{4} h^{4} E_{11}^{2}}{a^{6}}\left(\frac{\delta_{M S C B}^{2}}{P_{E N F}}\right) \times \\
& \frac{\left(f_{E B 2}+f_{T I M 2}+f_{F T 2}+f_{S-V 2}\right)}{\left(f_{E B 1}+f_{T I M 1}+f_{F T 1}+f_{S-V 1}\right)^{2}} \frac{1}{\left(9+f_{S H 2}\right)}, \\
& \frac{G_{I}}{G_{I I I}^{97.8 \%}}=\frac{b^{4} h^{4} E_{11}^{2}}{768 a^{6}}\left(\frac{\delta_{D C B}^{2}}{P_{M S C B}}\right) f_{I / I I I},
\end{aligned}
$$

where $f_{I / I I I}$ equals

$$
\frac{12+f_{W 2}+f_{T}+f_{S V}}{\left(1+\frac{f_{W 1}+f_{T}+\frac{f_{S V}}{2}}{4}\right)^{2}\left(f_{E B 2}+f_{T I M 2}+f_{F-T 2}+f_{S-V 2}\right)}
$$

In the followings the experimental work performed is detailed including separate pure mode-I, II, III and mixed-mode I/II, II/III, I/III and I/II/III tests. The measurement data are then reduced by the previously detailed improved beam theory scheme. In the final stage the fracture envelopes in the $\mathrm{G}_{I^{-}}-\mathrm{G}_{I I}, \mathrm{G}_{I I^{-}}$$\mathrm{G}_{I I I}, \mathrm{G}_{I}-\mathrm{G}_{I I I}$ planes and in the $\mathrm{G}_{I}-\mathrm{G}_{I I}-\mathrm{G}_{I I}$ space is presented for glass/polyester composite material.

\section{Experiments}

\subsection{Specimen manufacturing, materials}

The constituent materials of the investigated E-glass/polyester composite were procured from Novia Ltd. The properties of the E-glass fiber are $E=70 \mathrm{GPa}$ and $\nu=0.27$, while for the unsaturated polyester resin are $E=3.5 \mathrm{GPa}$ and $\nu=0.35$. Both were considered to be isotropic. The unidirectional $\left(\left[0^{\circ}\right]_{14}\right)$ E-glass/polyester specimens with nominal thickness of $2 h=6.2$ $\mathrm{mm}$, width of $b=9 \mathrm{~mm}$, and fiber-volume fraction of $V_{f}=43 \%$ were manufactured in a special pressure tool. A polyamide (PA) film with thickness of $0.03 \mathrm{~mm}$ was placed at the midplane of the specimens to make an artificial starting defect. A significant advantage of the present E-glass/polyester material is the transparency, which makes it possible to observe visually the crack initiation/propagation. The tool was left at room temperature until the specimens became dry. Then the specimens were removed from the tool and were further left at room temperature for 4-6 hours. At the final stage the specimens were cut to the desired length and were precracked in opening mode of 4$5 \mathrm{~mm}$ by using a sharp blade. The reason for that was that in this case it was possible to make a straight crack front, which is important in the case of the crack length measurement and the observation of the crack initiation. The slope of the loaddisplacement curves (i.e. the flexural modulus) was determined from a three-point bending test with span length of $2 L=150 \mathrm{~mm}$ using six uncracked specimens with thickness of $2 h=6.2 \mathrm{~mm}$ and width of $b=9 \mathrm{~mm}$. Then specimens were cut along the longitudinal direction in order to obtain very narrow specimens. The narrow specimens were rotated by $90^{\circ}$ about the longitudinal axis compared to the original measurements and the slope of the load-displacement data of the specimens was measured again. The flexural modulus was computed in accordance with simple beam theory expression. Both experiments resulted in $E_{11}=33$ $\mathrm{GPa}$, i.e. the material was found to be transversely isotropic. The additional properties were predicted from simple rules of mixture, in this way $E_{22}=E_{33}=7.2 \mathrm{GPa}, G_{12}=G_{13}=3 \mathrm{GPa}$ and $v_{12}=v_{13}=0.27$ were obtained.

\subsection{Double-cantilever beam test}

For the DCB test (Fig. 1b) four specimens with initial crack length of $a=55 \mathrm{~mm}$ were prepared. The specimens were tested using an Amsler testing machine and were loaded until the point of fracture initiation. At this point the critical crack opening displacement and the critical load were recorded. The displacement was measured using a mechanical dial gauge, while the values of the applied load were read from the scale of the testing machine. The ERR at the point of crack initiation was calculated by using Eq. (6), which resulted in $G_{I}=260.9 \mathrm{~J} / \mathrm{m}^{2}$.

\subsection{End-notched flexure test}

Similarly to the DCB test, four ENF coupons with initial crack length of $a=55 \mathrm{~mm}$ were prepared. The coupons were placed in a three-point bending setup with span length of $2 L=150 \mathrm{~mm}$ and were loaded up to fracture initiation in the same Amsler testing machine. At this point the critical load and displacement were recorded in a similar fashion to that mentioned in the DCB test. The ERR was calculated by the averaged experimental data using Eq. (12) and resulted in $G_{I I}=770.65$ $\mathrm{J} / \mathrm{m}^{2}$.

\subsection{Modified split-cantilever beam test}

For the MSCB measurements four specimens were prepared with $a=55 \mathrm{~mm}$ and $s=26 \mathrm{~mm}$. Each specimen was put into the loading rig shown in Fig. 2, the rig was adjusted in order to 
Fig. 3. The mixed-mode I/II/III DoublePrestressed End-notched Flexure specimen

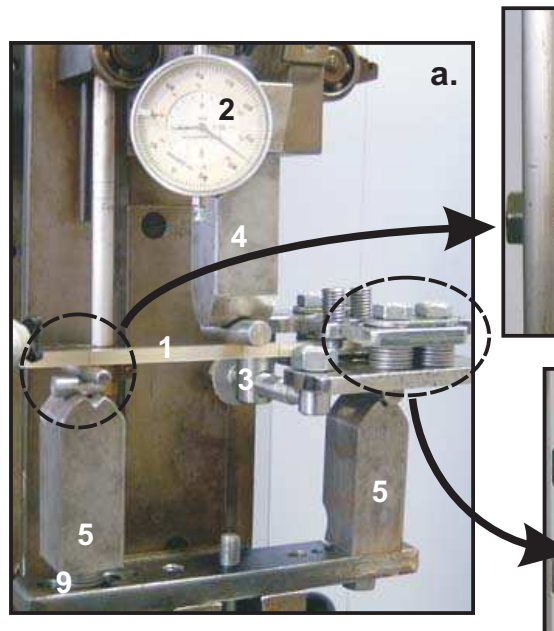

b.

1 Specimen

2 Dial gauge

3 Prestressing screw

4 Loading head

5 Supports

6 Steel roller

(support)

7 Steel roller

(prestress)

8 Grub screws

9 Underlays

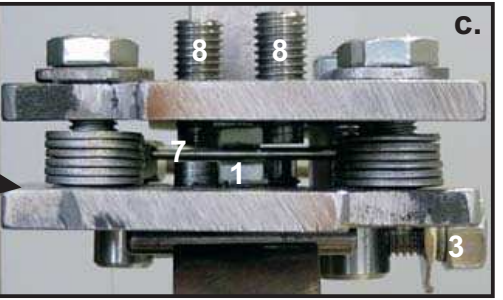

Fig. 4. The load-displacement curve of the $P E N F_{I / I I / I I I}$ (a) and the identification of crack initiation (b) (a)

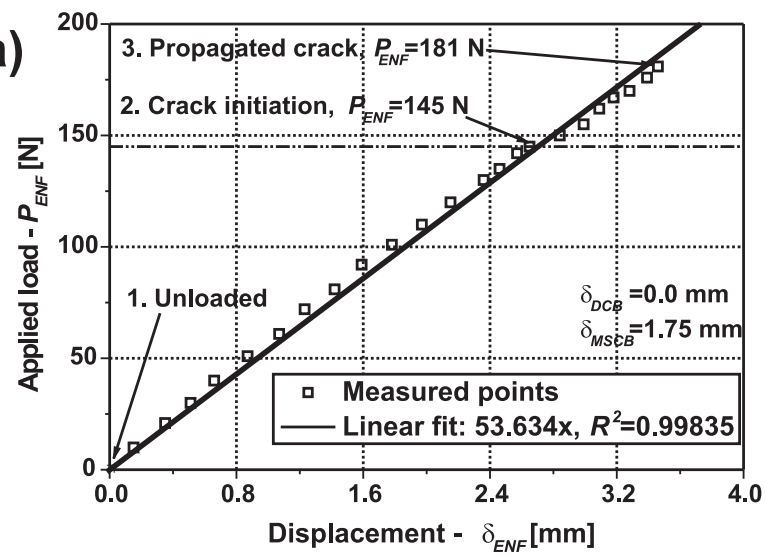

(b)

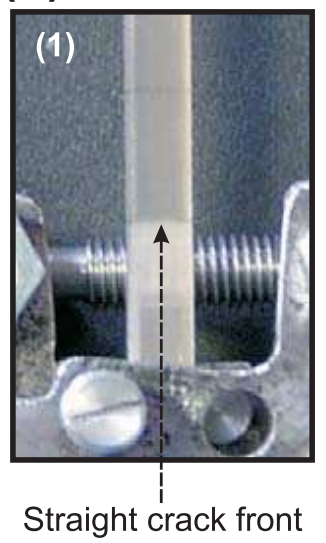

b) Displacement - $\delta_{\text {ENF }}[\mathrm{mm}]$

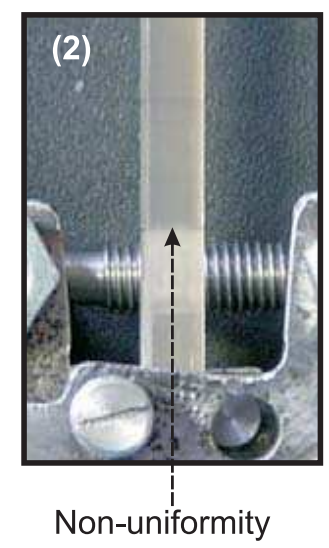

eliminate any play of the specimens. Then the specimens were loaded, the load and displacement values were read from the scale of the testing machine and using a mechanical dial gauge. The crack initiation was identified visually, so when the first non-uniformity in the previously straight crack front was observed it was believed to be the point of crack initiation. The ERR calculated by Eq. (20) is: $G_{I I I}=445.5 \mathrm{~J} / \mathrm{m}^{2}$.

\subsection{Prestressed end-notched flexure (I/I) test}

The PENF ${ }_{I / I I}$ test applies the common ENF test prestressed with a steel roller, in fact it is the superposition of the DCB and ENF specimens, see more details in [12]. The span length was
$2 L=150 \mathrm{~mm}$, the crack length of interest was $a=55 \mathrm{~mm}$. The reason for the latter was that the critical crack opening measured from the DCB test is about $4.5 \mathrm{~mm}$ (if $a=55 \mathrm{~mm}$ ) and the crack tip is far enough $(20 \mathrm{~mm})$ from the point of load application. The stiffness, the compliance and the mode-II ERR of the $\mathrm{PENF}_{I / I I}$ specimen are identical to those of the ENF specimen. We applied six steel rollers to control the mode-I part of the ERR including the following diameters: $d_{0}=1,1.5,2,2.4$, 3 and $4 \mathrm{~mm}$. It was assumed that the crack opening displacements $\left(\delta_{D C B}\right)$ in Eqs. (8) and (20) are equal to these values. Similarly to the DCB and ENF tests, we applied four coupons at each steel roller. The load-deflection data were measured by 
using the scale of the testing machine and the dial gauge (see Fig. 4). The measured data was reduced by using Eqs. (9) and (12) and six points in the $G_{I}-G_{I I}$ plane were obtained. These points were then fitted by two criteria functions: a criterion by Williams:

$$
\left(\frac{G_{I}}{G_{I C}}-1\right)\left(\frac{G_{I I}}{G_{I I C}}-1\right)-I_{i}\left(\frac{G_{I}}{G_{I C}}\right)\left(\frac{G_{I I}}{G_{I I C}}\right)=0
$$

where $G_{I C}$ is the critical mode-I ERR determined from the DCB test, $G_{I I C}$ is the mode-II critical ERR from the ENF test and $I_{i}$ is the interaction parameter between the modes and to be determined by a curve fit process. The another criterion - known as the power criterion - is:

$$
\left(\frac{G_{I}}{G_{I C}}\right)^{p_{1}}+\left(\frac{G_{I I}}{G_{I I C}}\right)^{p_{2}}=1
$$

where $p_{1}$ and $p_{2}$ can be determined by applying a proper curve fitting technique.

\subsection{Prestressed end-notched flexure (II/III) test}

The experimental equipment for the $\mathrm{PENF}_{I I / I I I}$ test is essentially the same as that in Fig. 3 without the prestressing steel roller. The test is the combination of the ENF and MSCB specimens. In a recent paper the details of the measurements have already been published [12]. The tests were carried out using an Amsler testing machine under displacement control. The span length was $2 L=150 \mathrm{~mm}$, the crack length of interest was $a=55$ $\mathrm{mm}$. The reason for the latter was (apart from the optimal case discussed in section 2) that the critical crack tearing measured from the MSCB test is about $2.5 \mathrm{~mm}$ (if $a=55 \mathrm{~mm}$ ) and the crack tip is far enough $(20 \mathrm{~mm})$ from the point of load application. The stiffness, the compliance and the mode-II ERR of the PENF specimen are identical to those of the ENF specimen. Six values of the crack tearing displacement (CTD), $\delta_{M S C B}$ were set using the prestressing screw in order to control the mode-III part of the total ERR: $\mathrm{MSCB}=0.875,1.313,1.750,2.023,2.188$ and 2.297 $\mathrm{mm}$. These values were calculated by being aware of the pitch of the prestressing screw. It was assumed that the crack tearing displacements $\left(\delta_{M S C B}\right)$ in Eqs. (25)-(29) are equal to these values. Similarly as in the MSCB and ENF tests, we applied four coupons at each steel roller. The load-deflection data were measured by using the scale of the testing machine and the dial gauge. The measured data were reduced by using Eqs. (12) and (26) and six points in the $G_{I I}-G_{I I I}$ plane were obtained, the points were fit by the same criteria as in the $\operatorname{PENF}_{I / I I}$ test, but $G_{I C}$ was replaced by $G_{I I C}$ and $G_{I I C}$ was replaced by $G_{I I I C}$, respectively.

\subsection{Prestressed split-cantilever beam (I/III) test}

The $\operatorname{PSCB}_{I / I I I}$ is also a brand new fracture mechanical test. In fact the PSCB ${ }_{I / I I I}$ test combines the mode-I DCB and the mode-III MSCB specimens. We insert a steel roller between the specimen arms and we put the prestressed specimen into the
MSCB rig in Fig. 2. The tests were carried out using an Amsler testing machine under displacement control. The crack length of interest was $a=55 \mathrm{~mm}$. The reason for the latter was (apart from the optimal case discussed in Section 2) that the critical crack opening displacement (COD) measured from the DCB test is about $4.5 \mathrm{~mm}$ (if $a=55 \mathrm{~mm}$ ) and the crack tip is far enough (29 $\mathrm{mm}$ ) from the point of the MSCB load application. It has already been shown that the stiffness, the compliance and the mode-III ERR of the PSCB I/III specimen are identical (with a very good approximation) to those of the MSCB specimen [12]. Six steel rollers were used including the following diameters: $d_{0}=1,1.5$, 2, 2.4, 3 and $4 \mathrm{~mm}$. It was assumed that the crack opening displacements $\left(\delta_{D C B}\right)$ in Eqs. (28) and (30) are identical to these values. It must be noted the the curved shape of the deflections induced by the steel rollers causes that the contact point between the roller and the specimen arm is slightly shifted. Considering the relatively small roller diameters it was estimated to be negligible. From other point of view the specimen arms transmitted a relatively high pressure to the steel roller, therefore the position of the rollers was always stable and no slip along the $x$ axis was observed during the measurements. Similarly to the previous tests, we applied four coupons at each steel roller. The load-deflection data was measured by using the scale of the testing machine and a mechanical dial gauge. In each case the critical load at crack initiation was determined. The measured data was reduced by using Eqs. (9) and (20) and six points in the $G_{I}-G_{I I I}$ plane were obtained, the fracture envelopes were determined by applying the power criterion and the one by Williams.

\subsection{Double-prestressed end-notched flexure (I/II/III) test}

The $\mathrm{PENF}_{I / I I / I I I}$ specimen (Fig. 1d) simply combines the mode-I DCB (Fig. 1a), the mode-II end-notched flexure (ENF) (Fig. 1b) and the modified split-cantilever beam (MSCB) specimens (Fig. 1c). The experimental equipment for the mixedmode I/II/III test is depicted in Fig. 3. The mode-I ERR is fixed by the steel roller (7), while the mode-III ERR of the system can be controlled by using the special grips of the MSCB test (steel blocks in Fig. 3). The mode-III load is transferred to the specimen through four grub screws (8), the crack tearing displacement $\left(\delta_{C T D}\right)$ is controlled by a prestressing screw (3). The critical crack tearing displacement of the MSCB specimen must be known in order not to cause crack initiation before testing. The double prestressed specimen is then put into a three-point bending fixture and the mode-II component of the ERR is increased by the external load. Using several steel rollers with different diameters and setting the crack tearing displacement to values below the critical CTD it is possible to provide any combination of the mode-I, mode-II and mode-III delamination fracture. The COD of system was varied including the following diameter values: $d_{0}=1,1.5,2,2.4,3$ and $4 \mathrm{~mm}$. The CTD of the specimen was set using the screw of the prestressing rig (see Fig. 3) including the values of: 0.875, 1.313, 1.75, 2.023 

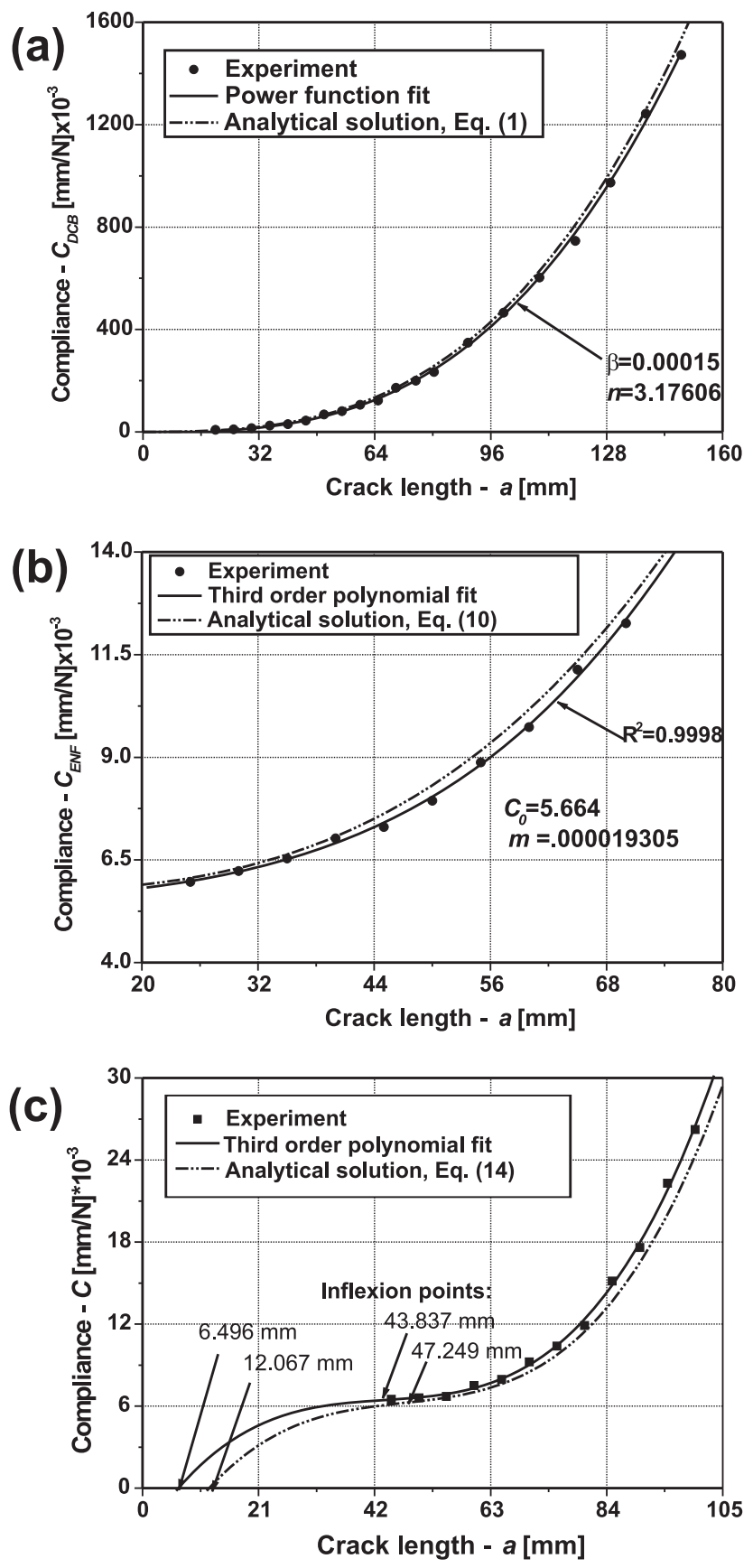

Fig. 5. Comparison of the analytical and experimental compliances of the DCB (a), ENF (b) and MSCB (c) specimens

2.188 and $2.297 \mathrm{~mm}$. The crack initiation was identified by photos. This process is shown in Fig. 4. The specimens were loaded subsequently, at some points, where the crack initiation was expected some of them was relieved and removed from the rig. The crack front was photographed and the specimen was put back to the rigs for further testing. At each prestressed state four coupons were used and the critical values of the load (PENF) were averaged. The measured load-displacement curves for the DCB, ENF, MSCB, $\mathrm{PENF}_{I / I I}, \mathrm{PENF}_{I I / I I I}, \mathrm{PSCB}_{I / I I I}$ and the $\mathrm{PENF}_{I / I I / I I I}$ specimens were found to be essentially linear. This confirms the application of the LEFM.
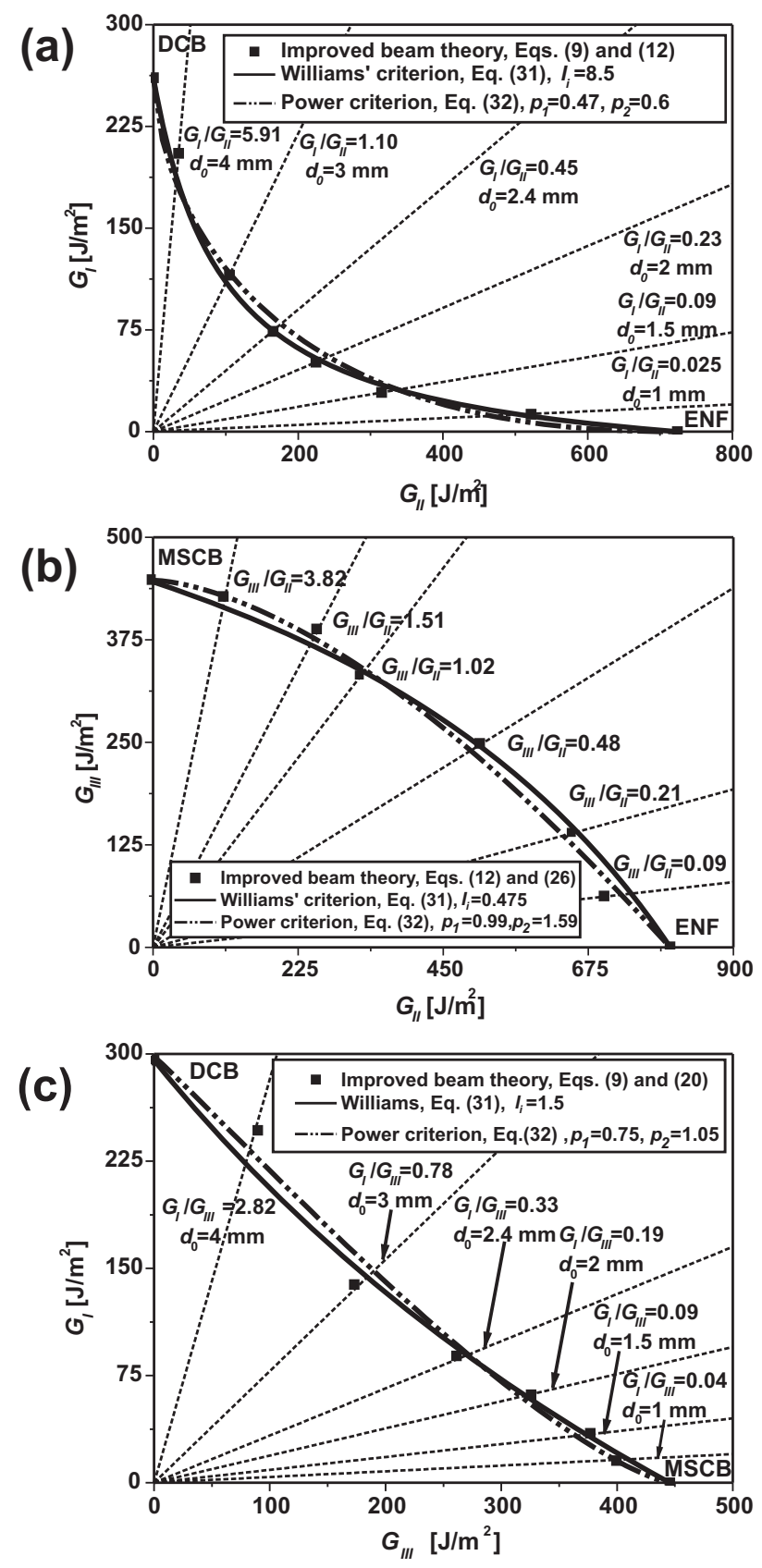

Fig. 6. Interlaminar fracture envelopes in the $G_{I}-G_{I I}$ (a), $G_{I I}-G_{I I I}$ (b) and $G_{I}-G_{I I I}$ (c) planes

\section{Data reduction}

The experimental data was reduced by using the improved beam theory expressions presented in Section 2. To confirm the accuracy of the analytical solutions the compliance of the DCB, ENF and MSCB specimens were determined in sufficiently extended crack length ranges. The measured points were fit by proper functions in each case: a power function in the case of the DCB $\left(C=\beta a^{n}\right)$, an incomplete third order polynomial in the case of the ENF specimen $\left(C=C_{0}+m a^{3}\right)$ and a full third order polynomial $\left(C=C_{0}+C_{1} a+C_{2} a^{2}+C_{3} a^{3}\right)$ in the MSCB specimen. The comparison between the fit curves and the analytical solution functions is demonstrated in Fig. 5. As it can be seen the agreement is very good, especially in the case of the 
DCB specimen. The relatively higher differences between the analytical and fit curves in the ENF and MSCB specimens can be explained by the lowest compliance and displacement values compared to the DCB test. Overall, the accuracy of the analytical solutions is excellent and it can be assumed that they provide the same accuracy if we apply them to data reduction in the prestressed specimens.
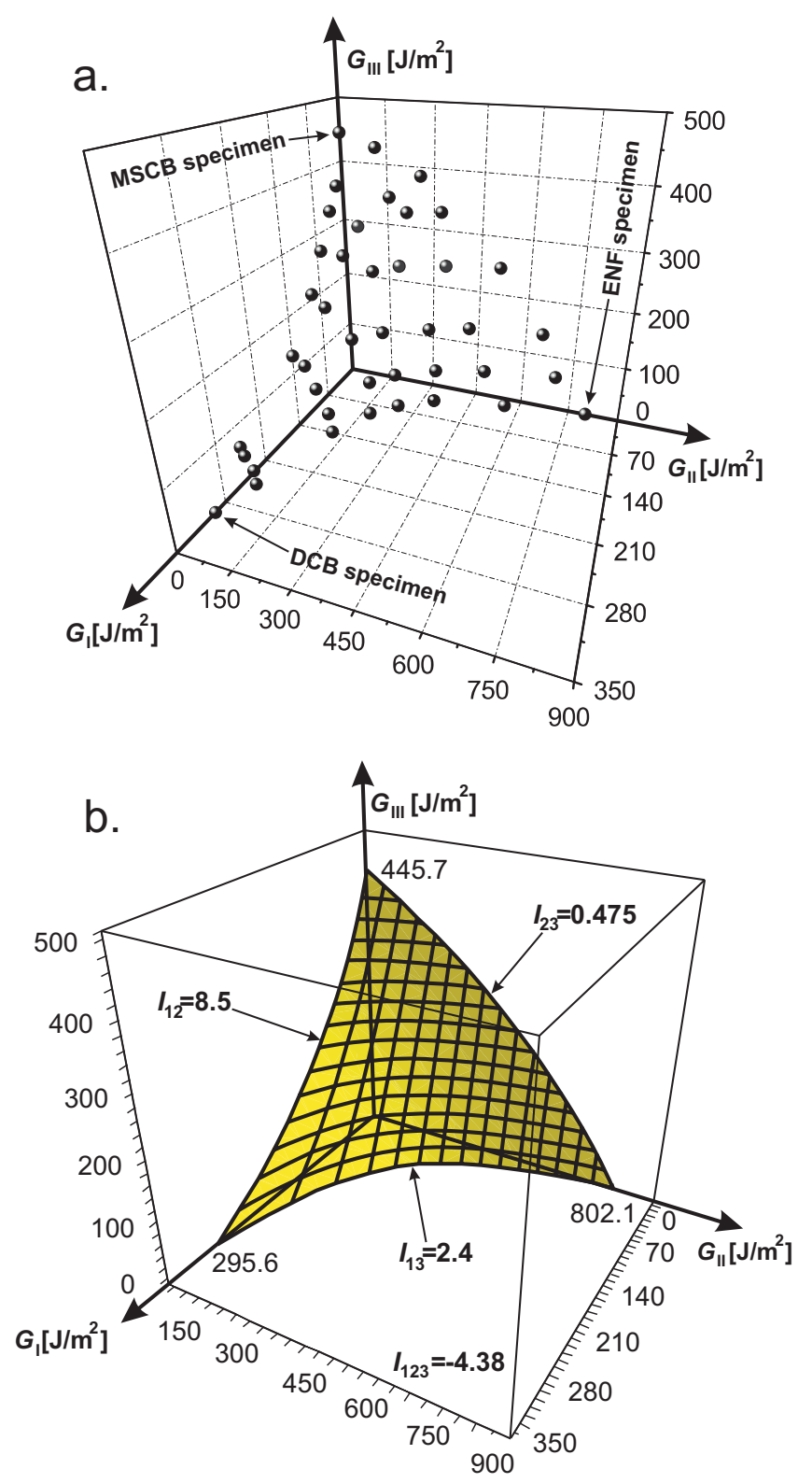

Fig. 7. The measured experimental points in the $G_{I}-G_{I I}-G_{I I I}$ space (a) and the calculated fracture surface of the material (b)

\subsection{Fracture envelopes}

The obtained fracture envelopes in the $G_{I}-G_{I I}, G_{I I}-G_{I I I}$ and the $G_{I}-G_{I I I}$ planes are shown in Fig. 6. The ones in the $G_{I}-G_{I I}$ and the $G_{I}-G_{I I I}$ planes have a concave nature, while the one in the $G_{I I}-G_{I I I}$ plane is convex. Overall, in each case there are significant interactions between the different fracture modes. To determine the delamination fracture surface of the present glass/polyester composite material we use the fracture envelopes in Fig. 6 and the measured data of the $\operatorname{PENF}_{I / I I / I I}$ specimen. We apply the following surface equation to display the fracture surface:

$$
\begin{gathered}
\frac{G_{I} G_{I I}}{G_{I C} G_{I I C}}\left(1-I_{12}\right)+\frac{G_{I I} G_{I I I}}{G_{I I C} G_{I I I C}}\left(1-I_{23}\right)+\frac{G_{I} G_{I I I}}{G_{I C} G_{I I I C}}\left(1-I_{13}\right) \\
-\frac{G_{I} G_{I I} G_{I I I}}{G_{I C} G_{I I C} G_{I I} I C} I_{123}-\frac{4}{3}\left(\frac{G_{I}}{G_{I C}}+\frac{G_{I I}}{G_{I I C}}+\frac{G_{I I I}}{G_{I I I C}}-1\right)=0,
\end{gathered}
$$

which is in fact the generalization of Williams' criterion for the $3 \mathrm{D}$ case. The interaction parameters $I_{12}, I_{23}$ and $I_{13}$ are known from $\mathrm{PENF}_{I / I I}, \mathrm{PENF}_{I I / I I I}$ and $\mathrm{PSCB}_{I / I I I}$ tests. So, there is only one unknown parameter, $I_{123}$ which can be determined using a curve fitting process. The measured points in the $G_{I}-G_{I I}-G_{I I I}$ space are shown in Fig. 7a and the fracture surface calculated from Eq. (33) is displayed in Fig. 7b. The interaction parameter is $I_{123}=-4.38$ indicating a significant interaction between the fracture modes.

\subsection{Conclusions}

In this work the concept of the prestressed delamination specimens were introduced, which can be applied to determine the fracture surface of laminated composite materials. In general the standard fracture mechanical tests cover only the mode-I, modeII and the mixed-mode I/II tests for crack initiation/propagation. In this work it was shown that for the complete fracture characterization of the material it is also reasonable to determine the fracture behaviour in the $G_{I I}-G_{I I I}$ and $G_{I}-G_{I I I}$ planes. The main advantage of the prestressed specimens are that the material can be tested at any mode ratio, the complete fracture space can be covered, the tests require relatively simple experimental equipment. The drawbacks of the test are that the mode ratio can not be designated before the testing process, because the mode ratio depends on the external load and also on the crack length.

\section{Acknowledgement}

This research work was sponsored by the Hungarian National Scientific Research Fund (OTKA) under Grant No. T34040-066 and by the János Bolyai Research Scolarship of the Hungarian Academy of Sciences.

\section{References}

1 Anderson TL, Fracture Mechanics - Fundamentals and Applications, CRC Press, Taylor and Francis Group, Boca Raton, London, New York, Singapore, 2005.

2 Hashemi S, Kinloch J, Williams JG, The effects of geometry, rate and temperature on mode I, mode II and mixed-mode I/II interlaminar fracture toughness of carbon-fibre/poly(ether-ether ketone) composites, Journal of Composite Materials 24 (1990), 918-956.

3 Carlsson LA, Gillespie JW, Pipes RB, On the analysis and design of the end notched flexure (ENF) specimen for mode II testing, Journal of Composite Materials 20 (1986), 594-604.

4 Lee SM, An edge crack torsion method for mode III delamination fracture testing, Journal of Composites Technology and Research 15 (1993), 193-201.

5 Reeder J.R., Crews Jr J.H., Mixed-mode bending method for delamination testing, AIAA Journal 28 (1990), 1270-1276. 
6 Davidson B.D, Sundararaman V, A single leg bending test for interfacial fracture toughness determination, International Journal of Fracture $\mathbf{7 8}$ (1996), 193-210.

7 Davies P, Ducept F, Brunner AJ, Blackman BRK, Morais de AB, Development of a standard mode II shear fracture test procedure, Proceedings of the 7th European Conference on Composite Materials (ECCM-7), London 2 (1996, May), 9-15.

8 Kamat SV, Srinivas M, Rao PR, Mixed mode I/III fracture toughness of armco iron, Acta Materialia 46 (14) (1998), 4985-4992.

9 Lazarus V, Leblond J-B, Mouschrif S-E, Crack front rotation and segmentation in mixed mode I+III or I+II+III. Part II: Comparison with experiments, Journal of the Mechanics and Physiscs of Solids 49 (2001), 1421-1443.

$10 \mathbf{~ L i ~ H , ~ J o n e s ~ R H , ~ H i r t h ~ J P , ~ M i x e d ~ m o d e ~ I / I I I ~ f r a c t u r e ~ t o u g h n e s s ~ o f ~ a ~ V - 5 C r - ~}$ 5Ti alloy at 100 C, Scripta Metallurgica et Materialia 32 (4) (1995), 611-616.

11 Szekrényes A, Prestressed fracture specimen for delamination testing of composites, International Journal of Fracture 139 (2006), 213-237.

12 , Delamination fracture analysis in the $G_{I}-G_{I I I}$ plane using prestressed transparent composite beams, International Journal of Solids and Structures 44 (2007), 3359-3378.

13 Sharif F, Kortschot MT, Martin RH, Mode III Delamination Using a Split Cantilever Beam, Composite Materials: Fatigue and Fracture - Fifth Volume ASTM STP, ASTM, Philadelphia, Edited by Martin R.H. 1230 (1995), 8599.

14 Cicci D, Sharif F, Kortschot MT, Data reduction for the split cantilever beam mode III delamination test, Proceedings of ACCM 10, Whistler, British Columbia, Canada (1995, 14/18 August).

15 Szekrényes A, Interlaminar fracture analysis in the $G_{I}-G_{I I I}$ plane using prestressed transparent composite beams, ready to submission (2008).

16 _ Improved analysis of unidirectional composite delamination specimens, Mechanics of Materials 39 (2007), 953-974. 